\title{
2D-Based 3D Volume Retrieval Using Singular Value Decomposition of Detected Regions
}

\author{
Alba G. Seco de Herrera ${ }^{1}$, Antonio Foncubierta-Rodríguez ${ }^{1}$, \\ Emanuele Schiavi ${ }^{2}$, and Henning Müller ${ }^{1}$ \\ ${ }^{1}$ University of Applied Sciences Western Switzerland (HES-SO), \\ ${ }^{2}$ University Rey Juan Carlos, Spain. \\ alba.garcia@hevs.ch
}

\begin{abstract}
In this paper, a novel 3D retrieval model to retrieve medical volumes using $2 \mathrm{D}$ images as input is proposed. The main idea consists of applying a multi-scale detection of saliency of image regions. Then, the $3 \mathrm{D}$ volumes with the regions for each of the scales are associated with a set of projections onto the three canonical planes. The 3D shape is indirectly represented by a $2 \mathrm{D}$-shape descriptor so that the $3 \mathrm{D}$-shape matching is transformed into measuring similarity between $2 \mathrm{D}$-shapes. The shape descriptor is defined by the set of the $k$ largest singular values of the 2D images and Euclidean distance between the vector descriptors is used as a similarity measure. The preliminary results obtained on a simple database show promising performance with a mean average precision (MAP) of 0.82 and could allow using the approach as part of a retrieval system in clinical routine.
\end{abstract}

Keywords: 2D-based 3D retrieval, region detector, singular value decomposition

\section{Introduction}

Radiologists are dealing with an increasing number and also a strongly increasing variety of medical images $[1,2]$. Imaging techniques are an essential part of diagnosis and treatment planning. Many physicians also have regular information needs during clinical work, teaching preparation and research activities $[3,4]$, where computerized tools can help make the search more efficient and effective. Medical image retrieval has been an area of intensive research for the past 20 years $[2,5]$ and 3D data access has started to get increasing attention, as it is the medical imaging modality that is increasing fastest in terms of data produced. The need to search for $3 \mathrm{D}$ volumes using their visual content to complement textual search in annotations has led to the development of several approaches to compute similarity between two 3D volumes in recent years [6]. Users can search for 3D volumes by supplying an example query volume or mark a volume of interest. Several articles describe these types of image search [7]. In this paper, we propose a novel 3D retrieval model to retrieve medical volumes using $2 \mathrm{D}$ images as input. This is intuitive as $2 \mathrm{D}$ images might be available easily 
as a starting point for queries, for example via images in the literature, images used in scientific presentations or for teaching. Medical image databases often contain images of many different modalities, taken under varied conditions [8]. The 2D-based 3D retrieval also provides numerous opportunities for working between 2D modalities such as $\mathrm{x}$-ray and 3D modalities such as CT. However, many current $2 \mathrm{D}$ approaches to search for $3 \mathrm{D}$ volumes are based on retrieval by sketch $[6,9,10]$. Most applications of $2 \mathrm{D}$-based $3 \mathrm{D}$ volume retrieval focus on objects $[11,12]$ and not on medical images where generally solid texture and not an object surface is the target for search.

When retrieving $3 \mathrm{D}$ volumes using $2 \mathrm{D}$ query images that are not necessarily single slices of a volume, we need to compute the distance between the query image and the $3 \mathrm{D}$ volumes in the database. The algorithm we propose is composed of four stages:

- region detection in 3D volumes;

- volume projection onto 2D planes;

- singular value extraction from the projections;

- similarity calculation between a query and the projections.

Conventionally, regions of interest for retrieval are detected using algorithms such as the Sobel filter, Prewitt algorithm or Laplacian of Gaussian [13]. In the first step of our approach, a region of interest detector for medical images is applied that is able to provide locally salient regions at various scales [7].

The binary 3D images with the regions detected are then associated with three canonical 2D projections at each of the scales. A few 3D retrieval approaches using $2 \mathrm{D}$ projection views have also been proposed recently [11,12]. Most of them use polyhedron-based views [14] for the projections. In the approach presented in this paper canonical projections are used because medical images are usually created using very standardized acquisition settings. As a result we obtain the $2 \mathrm{D}$ silhouette views of the volumes. We then have a simpler binary 2D silhouette retrieval problem. To compute the distance between two views, we need to extract an appropriate shape descriptor. Each image is processed in order to extract and describe the shape of the regions. The shape descriptor we chose is defined by the set of the $k$ largest singular values of the $2 \mathrm{D}$ regions. In the literature, singular values were used in the past as shape descriptors for face recognition [15-17]. In order to validate the approach we use a simple database consisting of head and thorax volumes. The Euclidean norm between the shape descriptors is used as similarity measure.

The rest of the paper is organized as follows. In Section 2 the database and the methods used on our approach are described. The results are presented in Section 3. Section 4 concludes the paper and list several future research ideas.

\section{Materials and Methods}

This section describes the materials and methods used for 2D-based 3D medical image retrieval (see also Figure 1). Let $I_{v} \in \mathcal{M}_{m \times n \times o}(\mathbb{R})$ be a volume that is part of the database. The volumes are analyzed as follows: 


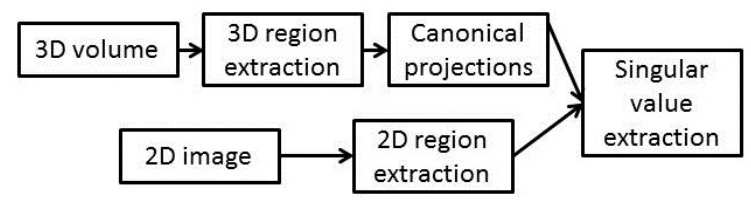

Fig. 1. Schematic overview of the image processing steps of the described approach.

1. A volume $I_{v_{b r}} \in \mathcal{M}_{m \times n \times o}(\{0,1\})$ with detected binary regions of $I_{v}$ is created (see subsection 2.2 for more details).

2. $I_{v_{b r}}$ is projected onto the three canonical axes, $P_{x}, P_{y}$ and $P_{z}$ (see subsection 2.3).

3. The $k$ largest singular values of the projections $P_{x}, P_{y}$ and $P_{z}$ are used as shape descriptors (see subsection 2.4).

Let $I \in \mathcal{M}_{p \times q}(\mathbb{R})$ be a $2 \mathrm{D}$ image that can be used as an example query to find corresponding volumes. The following steps are applied to this image:

1. An image $I_{b r} \in \mathcal{M}_{p \times q}(\{0,1\})$ with detected binary regions $I$ is created (see subsection 2.2).

2. The $k$ largest singular values of the image with the detected regions $I_{b r}$ are used as shape descriptor (see subsection 2.4).

Finally, the distance between the singular values of the 2D and of each of the three projections of the $3 \mathrm{D}$ volume are calculated. Only the nearest projection is used for the retrieval step (see subsection 2.5). In the following subsections the steps are described in more detail.

\subsection{Database}

To carry out this preliminary study and show the feasibility of the approach, images from three existing databases are used (two databases to create a set of volumes and one database to get $2 \mathrm{D}$ query images). All the volumes of the two databases are images taken for research from clinical routine:

- 41 T1 and T2 weighted head MRI series were used. All images were acquired on a 3T MR imaging scanner (Magnetom Trio a Tim System, Siemens, Germany) using a head coil.

- 41 thorax CTs were used, acquired on a GE DECT (General Electrics Dual Energy Computed Tomography) scanner; only one energy level was used.

$2 \mathrm{D}$ images from the same anatomic regions as the selected $3 \mathrm{D}$ volumes (head, chest) were chosen from the publicly available ImageCLEF medical database [18] as query images. This database consists of images from articles of the biomedical open access literature from various modalities (CT and MRI slices, x-ray images, etc.). Since the images belong to scientific articles many of them contain annotations such as colored arrows or are cropped and modified in many other 

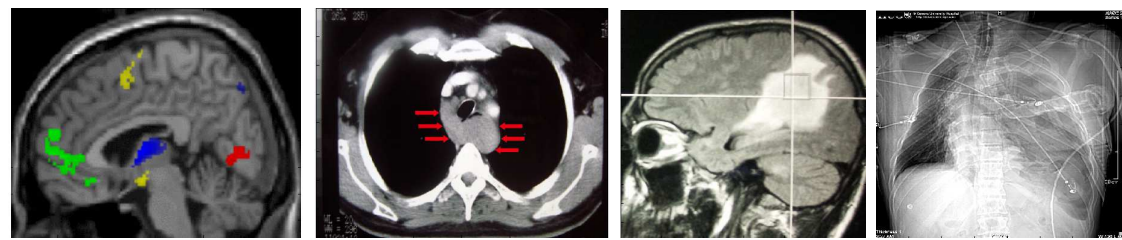

Fig. 2. Sample images used from ImageCLEF data set.

ways (see Fig. 2). Images can be of different modalities than the volumes for the same anatomic region and in differing level/window settings. In total, $952 \mathrm{D}$ query images were used ( 47 of the head and 48 of the thorax). The $2 \mathrm{D}$ database was divided into two subsets, a training set to optimize the parameters (24 of the head and 24 of the thorax) and a test set for showing the stability of the approach (23 of the head and 24 of the thorax). The query images contain also other modalities than the volumes, for example chest $\mathrm{x}$-rays to search for chest CT volumes.

\subsection{Region Detection}

A key-region detector [7] is applied providing locally relevant regions of interest based on the actual patterns of the image with no predefined shape. The keyregion detector is based on a wavelet pyramid, providing meaningful regions at various scales. We can use the same algorithm to detect regions in $3 \mathrm{D}$ as well as in $2 \mathrm{D}$ (see Fig. 3) due to the dimensionality-independence of the detector. The algorithm automatically detects salient regions at various scales. Two examples showing the regions detected in 2D images are illustrated in Fig. 3. Fig. 4(d) shows the regions detected in a volume.
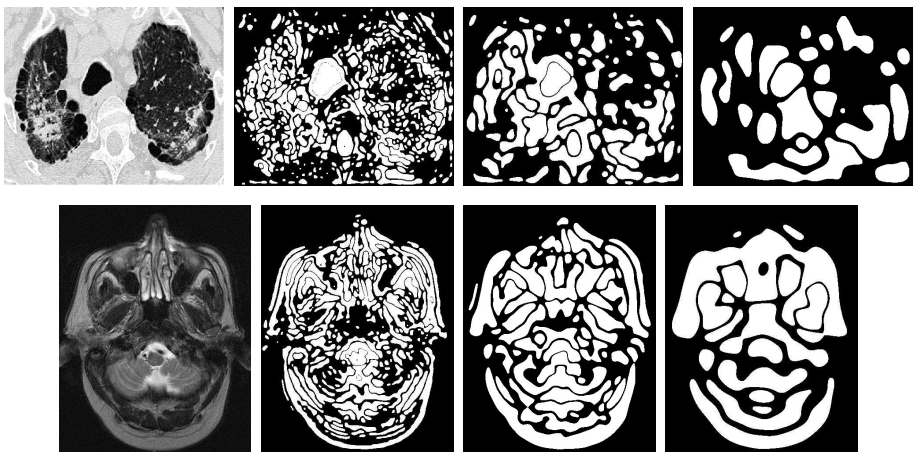

Fig. 3. 2D images and their detected regions at the scales 4,8 and 16 


\section{$2.3 \quad$ Projections}

The projection step aims at extracting a 2D representation of the binary $3 \mathrm{D}$ volumes to be able to compare them with the $2 \mathrm{D}$ queries. To compute the view of a $3 \mathrm{D}$ volume on a plain, we extract the projections along the three canonical axes $(x, y$ and $z)$ [19]. Canonical projections are used as the acquisition protocol of the medical volumes is fully standardized and usually has these same projections. The projections of a binary region image $I_{v_{b r}}(x, y, z) \in \mathcal{M}_{m \times n \times o}(\{0,1\})$ are (see Fig. 4(a), 4(b) and 4(c)):

$$
\begin{aligned}
& P_{x}\left(y_{0}, z_{0}\right)=\left\{\begin{array}{l}
1 \text { if } \exists x / I_{v_{b r}}\left(x, y_{0}, z_{0}\right)=1 \\
0 \text { otherwise }
\end{array} \text {,for } 0<y_{0}<N, 0<z_{0}<O\right. \\
& P_{y}\left(x_{0}, z_{0}\right)=\left\{\begin{array}{l}
1 \text { if } \exists y / I_{v_{b r}}\left(x_{0}, y, z_{0}\right)=1 \\
0 \text { otherwise }
\end{array}, \text { for } 0<x_{0}<M, 0<z_{0}<O\right. \\
& P_{z}\left(x_{0}, y_{0}\right)=\left\{\begin{array}{l}
1 \text { if } \exists z / I_{v_{b r}}\left(x_{0}, y_{0}, z\right)=1 \\
0 \text { otherwise }
\end{array}, \text { for } 0<x_{0}<M, 0<y_{0}<N\right.
\end{aligned}
$$

\subsection{Singular Value Decomposition}

The singular value decomposition (SVD) of a rectangular matrix $A \in \mathcal{M}_{m \times n}(\mathbb{R})$ is decomposed in the form [20]:

$$
A=U D V^{T}
$$

where $U \in \mathcal{M}_{m \times m}(\mathbb{R})$ and $V \in \mathcal{M}_{n \times n}(\mathbb{R})$ are orthogonal matrices. The singular values of $A, \sigma_{1} \geq \sigma_{2}, \ldots \geq \sigma_{p} \geq 0$ with $p=\min \{m, n\}$, appear in descending order along the main diagonal $D$.

By applying a singular value decomposition, the matrix can be decomposed into a matrix that contains intrinsic shape information, the singular value matrix $D$, and matrices with information about corresponding points $U$ and $V$. An important property of the SVD is that the largest singular values in $D$ always hold the maximum possible information and show stability for most image modifications [21] because of its geometrical invariance [21]. We use a set of $k$ largest singular values of the binary images as a shape descriptor. The truncated SVD captures most of the underlying structure and at the same time removes noise [22].

\subsection{Comparison}

We measure the effectiveness of our approach using 2D images as queries and $3 \mathrm{D}$ volumes as the database and anatomic region as the ground truth. To assess the overall performance of our algorithm, we test our retrieval algorithm on the complete training query set. The parameters which achieved the best results over the training data were used for evaluation on the test data. Separation between test and training data set is random, respecting that both contain an 

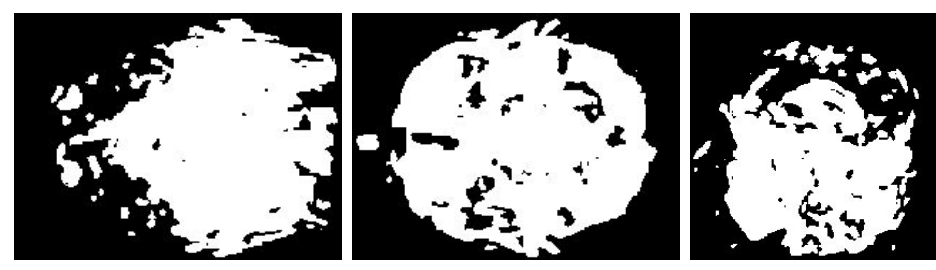

(a) scale 4
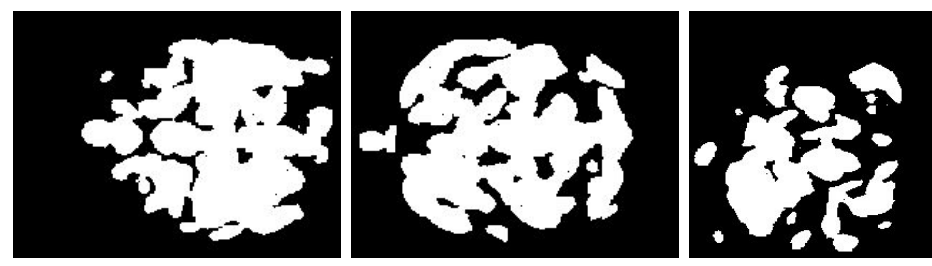

(b) scale 8
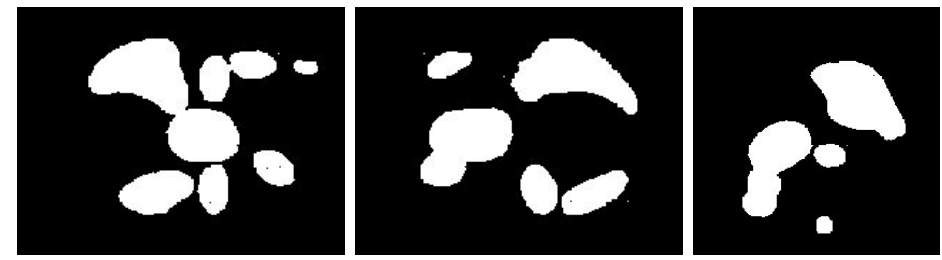

(c) scale 16

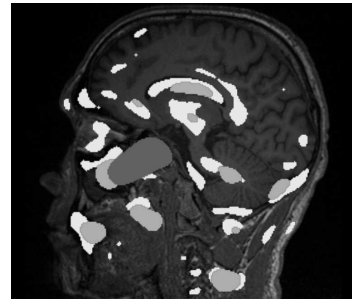

(d) Overlapping regions on the original volume at scales 4,8 and 16 .

Fig. 4. 2D binary projections at various scales; original volume with regions detected. 
equal number of instances from each anatomic region. In order to measure the quality of the retrieval, the average precision (AveP) is calculated for each query to get an overall picture of the quality in such a simple retrieval scenario. Then, the mean value of the average precision scores from all the queries is calculated (MAP) [23]. There are many evaluation methods proposed in image retrieval to evaluate the effectiveness of the systems [24]. MAP was chosen as it is a standard measure in the main information retrieval benchmarks [25]. MAP scores were also measured for various similarity distances over the training set.

\section{$3 \quad$ Results}

We implement a 2D-based 3D volume retrieval system based on the proposed framework. The subjects of the $2 \mathrm{D}$ views are not the same as the $3 \mathrm{D}$ volumes and the task is harder as the views can vary strongly and can be cropped and modified in many ways. The performance of this scenario is measured by the MAP of the volumes retrieved when 2D images are taken as queries. For our experiments over the test set the 10 largest singular values from the scale 4 were used. These parameters were taken from an analysis on the training set. The approach achieves a MAP of $\mathbf{0 . 8 2 3 6}$ on the test data, showing the stability of the approach. In the analysis over the training set we tested various similarity measures: Euclidean distance (Table 1), Canberra distance (Table 2), chi-square distance (Table 3), cosine similarity (Table 4), histogram intersection (Table 5) and Jeffrey divergence (Table 6). The results show that using the Euclidean distance the best results are achieved. For this reason Euclidean distance was used to evaluate MAP over the test data.

Table 1. MAP using various scales and the $k$ largest singular values on the training set using Euclidean distance.

\begin{tabular}{lccccccc} 
& $k$ & 3 & 5 & 10 & 15 & 25 & 50 \\
scale & & & & & & & \\
\hline 2 & 0.5264 & 0.5655 & 0.6574 & 0.6775 & 0.6645 & 0.6518 \\
4 & & 0.6623 & 0.7251 & $\mathbf{0 . 7 6 4 6}$ & 0.7150 & 0.6462 & 0.6478 \\
8 & & 0.6153 & 0.6116 & 0.59011 & 0.5814 & 0.5767 & 0.5656 \\
16 & & 0.5310 & 0.5405 & 0.5503 & 0.5579 & 0.5712 & 0.5875 \\
32 & & 0.5598 & 0.5696 & 0.5585 & 0.5529 & 0.5578 & 0.5634 \\
64 & & 0.6893 & 0.6894 & 0.6894 & 0.6894 & 0.6894 & 0.6894 \\
\hline
\end{tabular}

\section{Conclusions and Future Work}

This paper describes an approach for 2D-based 3D retrieval in medical databases. Such a system can be useful for clinicians searching for volumes when they have a 
Table 2. MAP using various scales and the $k$ largest singular values on the training set using Canberra distance.

\begin{tabular}{lccccccc} 
& $k$ & 3 & 5 & 10 & 15 & 25 & 50 \\
\hline 2 & & & & & & & \\
\hline 2 & & 0.5403 & 0.5841 & 0.6606 & 0.6906 & 0.7048 & 0.6514 \\
4 & & 0.6360 & 0.6547 & 0.6653 & 0.6691 & 0.6652 & 0.6599 \\
8 & & 0.6179 & 0.6367 & 0.6149 & 0.6248 & 0.6394 & 0.6432 \\
16 & & 0.5606 & 0.5493 & 0.5399 & 0.5598 & 0.5828 & 0.6137 \\
32 & & 0.5578 & 0.5519 & 0.5555 & 0.5480 & 0.6151 & 0.6326 \\
64 & & $\mathbf{0 . 7 0 7 3}$ & 0.6895 & 0.6621 & 0.6361 & 0.5897 & 0.5897 \\
\hline
\end{tabular}

Table 3. MAP using various scales and the $k$ largest singular values on the training set using chi-square distance.

\begin{tabular}{lccccccc} 
& $k$ & 3 & 5 & 10 & 15 & 25 & 50 \\
\hline scale & & & & & & & \\
\hline 2 & & 0.5390 & 0.5831 & 0.6839 & 0.6790 & 0.6490 & 0.6523 \\
4 & & 0.6422 & 0.6761 & $\mathbf{0 . 7 5 1 1}$ & 0.7278 & 0.6632 & 0.650 \\
8 & & 0.6126 & 0.6180 & 0.6012 & 0.5972 & 0.5883 & 0.5814 \\
16 & & 0.5456 & 0.5434 & 0.5536 & 0.5519 & 0.5495 & 0.5707 \\
32 & & 0.5630 & 0.6126 & 0.5904 & 0.5582 & 0.5674 & 0.5644 \\
64 & & 0.6881 & 0.6881 & 0.6881 & 0.6881 & 0.6881 & 0.6881 \\
\hline
\end{tabular}

Table 4. MAP using various scales and the $k$ largest singular values on the training set using cosine similarity.

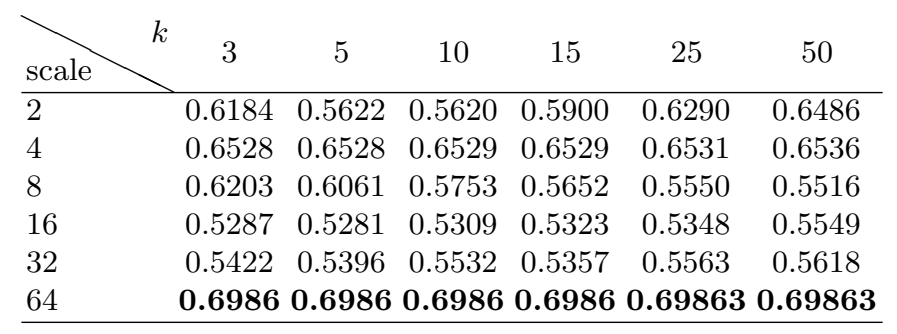

Table 5. MAP using various scales and the $k$ largest singular values on the training set using histogram intersection.

\begin{tabular}{lcccccc} 
& $k$ & & & & & \\
scale & 3 & 5 & 10 & 15 & 25 & 50 \\
\hline 2 & 0.5291 & 0.5718 & 0.6659 & 0.6821 & 0.6615 & 0.6527 \\
4 & 0.6388 & 0.7146 & $\mathbf{0 . 7 5 1 2}$ & 0.7249 & 0.6491 & 0.6533 \\
8 & 0.5966 & 0.6141 & 0.5964 & 0.5782 & 0.5696 & 0.5618 \\
16 & 0.5575 & 0.5633 & 0.5812 & 0.5973 & 0.6096 & 0.6106 \\
32 & 0.5512 & 0.5553 & 0.5634 & 0.5738 & 0.5671 & 0.5723 \\
64 & 0.6986 & 0.69863 & 0.69863 & 0.69863 & 0.69863 & 0.69863 \\
\hline
\end{tabular}


Table 6. MAP using various scales and the $k$ largest singular values on the training set using Jeffrey divergence.

\begin{tabular}{|c|c|c|c|c|c|c|}
\hline & 3 & 5 & 10 & 15 & 25 & 50 \\
\hline 2 & 0.5531 & 0.5921 & 0.6934 & 0.6789 & 0.6565 & 0.6565 \\
\hline 4 & 0.642 & 0.6786 & 0.7519 & 0.7260 & 0.6565 & 0.6565 \\
\hline 8 & 0.617 & 0.6234 & 0.6029 & 0.6595 & 0.6565 & 0.6565 \\
\hline 16 & 0.544 & 0.5406 & 0.5702 & 0.6319 & 0.6565 & 0.6565 \\
\hline 32 & 0.575 & 0.6211 & 0.6082 & 0.6553 & 0.6565 & 0.6565 \\
\hline 64 & 0.689 & 0.6894 & 0.6894 & 0.6894 & 0.6894 & 0.6894 \\
\hline
\end{tabular}

single 2D view of an image available, for example from a 2D modality, a medical article or from a PowerPoint presentation.

The preliminary results obtained show promising performance with a MAP of 0.82 and allow using the approach as part of a retrieval system for clinical routine. This shows that most images of the same anatomic region can be identified even when queries are not of the same modality or are cropped and otherwise modified. Using 2D images as queries to retrieve $3 \mathrm{D}$ volumes may provide a useful tool for radiologists searching for information on specific regions of interest. Obviously, the current scenario of using only two anatomic regions is a very simplified scenario. It is planned to extend the approach to a much larger set of anatomic regions. Larger databases are currently being created and should be directly usable with the same approach. In terms of relevance for specific information needs it is also clear that not only anatomy are important but also local characteristics representing pathologies. Still the described approach already allows filtering out similar anatomic regions that can then be further exploited for visually similar regions of interest. The approach is a step towards retrieval between images of differing dimensionality.

\section{Acknowledgments}

The research leading to these results has received funding from the European Union's Seventh Framework Programme under grant agreement 257528 (KHRESMOI) and 258191 (PROMISE).

\section{References}

1. Akgül, C., Rubin, D., Napel, S., Beaulieu, C., Greenspan, H., Acar, B.: Contentbased image retrieval in radiology: Current status and future directions. Journal of Digital Imaging 24(2) (2011) 208-222

2. Hwang Kyung, H., Lee, H., Choi, D.: Medical image retrieval: Past and present. Health Information Research 18(1) (2012) 3-9 
3. Hersh, W., Jensen, J., Müller, H., Gorman, P., Ruch, P.: A qualitative task analysis for developing an image retrieval test collection. In: ImageCLEF/MUSCLE workshop on image retrieval evaluation, Vienna, Austria (2005) 11-16

4. Müller, H., Despont-Gros, C., Hersh, W., Jensen, J., Lovis, C., Geissbuhler, A.: Health care professionals' image use and search behaviour. In: Proceedings of the Medical Informatics Europe Conference (MIE 2006). IOS Press, Studies in Health Technology and Informatics, Maastricht, The Netherlands (August 2006) 24-32

5. Müller, H., Michoux, N., Bandon, D., Geissbuhler, A.: A review of content-based image retrieval systems in medicine-clinical benefits and future directions. International Journal of Medical Informatics 73(1) (2004) 1-23

6. Yoon, S.M., Scherer, M., Schreck, T., Kuijper, A.: Sketch-based 3D model retrieval using diffusion tensor fields of suggestive contours. In: ACM Multimedia. (2010) $193-200$

7. Foncubierta-Rodríguez, A., Müller, H., Depeursinge, A.: Region-based volumetric medical image retrieval. In: SPIE Medical Imaging: Advanced PACS-based Imaging Informatics and Therapeutic Applications. (2013)

8. Kalpathy-Cramer, J., Hersh, W.: Medical image retrieval and automatic annotation: OHSU at ImageCLEF 2007. In: Working Notes of the 2007 CLEF Workshop, Budapest, Hungary (September 2007)

9. $\mathrm{Pu}$, J., Ramani, K.: A 3D model retrieval method using 2D freehand sketches. In: Emory University. (2005) 22-25

10. Li, B., Johan, H.: Sketch-based 3D model retrieval by incorporating 2D-3D alignment. Multimedia Tools and Applications (2012) 1-23

11. Napoléon, T., Sahbi, H.: Content-based 3D object retrieval using 2D views. In: Proceedings of the International Conference on Image Processing. ICIP'09 (2009) $1437-1440$

12. Petre, R.D., Zaharia, T., Prêteux, F.: An overview of view-based 2D/3D indexing methods. In: SPIE Mathematics of Data/Image Coding, Compression, and Encryption with Applications XII. Volume 7799. (2010)

13. Yu-qian, Z., Wei-hua, G., Zhen-cheng, C., Jing-tian, T., Ling-yun, L.: Medical images edge detection based on mathematical morphology. In: Proceedings of the 2005 IEEE Engineering in Medicine and Biology 27th Annual Conference. (2005)

14. Xiao, H., Zhang, X.: A method for content-based 3D model retrieval by 2D projection views. WSEAS Transactions on Circuits and Systems 7(5) (May 2008) 445-449

15. Smeets, D., Fabry, T., Hermans, J., Vandermeulen, D., Suetens, P.: Isometric deformation modeling using singular value decomposition for 3D expression-invariant face recognition. In: IEEE 3rd International Conference on Biometrics: Theory, Applications and Systems. BTAS '09 (2009) 1-6

16. Smeets, D., Hermans, J., Vandermeulen, D., Suetens, P.: Isometric deformation invariant 3D shape recognition. Pattern Recognition 45(7) (2012) 2817-2831

17. Wang, Y., Wang, Y., Jain, A.K., Tan, T.: Face verification based on bagging rbf networks. In: Proceedings of the 2006 international conference on Advances in Biometrics. ICB'06, Berlin, Heidelberg, Springer-Verlag (2006) 69-77

18. Müller, H., García Seco de Herrera, A., Kalpathy-Cramer, J., Demner Fushman, D., Antani, S., Eggel, I.: Overview of the ImageCLEF 2012 medical image retrieval and classi?cation tasks. In: Working Notes of CLEF 2012 (Cross Language Evaluation Forum). (September 2012)

19. Lehmann, G.: Image projections along an axis. The Insight Journal (2006)

20. Golub, G.H., van Loan, C.F.: Matrix Computations. (1996) 
21. Jiao, Y., Yang, B., Wang, H., Niu, X.: SVD based robust image content retrieval. In: Proceedings of the 2006 International Conference on Intelligent Information Hiding and Multimedia. IIH-MSP '06 (2006) 351-354

22. Berry, M.W., Dumais, S.T., O'Brien, G.W.: Using linear algebra for intelligent information retrieval. SIAM Review 37(4) (December 1995) 573-595

23. Kishida, K.: Property of average precision and its generalization: An examination of evaluation indicator for information retrieval experiments. technical report, National Institute of Informatics, Tokyo, Japan (2005)

24. Volk, M., Ripplinger, B., Vintar, S., Buitelaar, P., Raileanu, D., Sacaleanu, B.: Semantic annotation for concept-based cross-language medical information retrieval. International Journal of Medical Informatics 67 (2002) 97-112

25. Voorhees, E.M., Harman, D.K.: TREC: Experiment and evaluation in information retrieval. MIT press Cambridge (2005) 